\title{
BMJ Open Nutritional impact on Immunological maturation during Childhood in relation to the Environment (NICE): a prospective birth cohort in northern Sweden
}

\author{
Malin Barman, ${ }^{1,2}$ Fiona Murray, ${ }^{3}$ Angelina I Bernardi, ${ }^{4}$ Karin Broberg, ${ }^{5}$ Sven Bölte, ${ }^{6}$ \\ Bill Hesselmar, ${ }^{7}$ Bo Jacobsson, ${ }^{2}$ Karin Jonsson, ${ }^{1}$ Maria Kippler, ${ }^{5}$ Hardis Rabe, ${ }^{4}$ \\ Alastair B Ross, ${ }^{1}$ Fei Sjöberg, ${ }^{4}$ Nicklas Strömberg, ${ }^{8}$ Marie Vahter, ${ }^{5}$ Agnes E Wold, ${ }^{4}$ \\ Ann-Sofie Sandberg, ${ }^{1}$ Anna Sandin ${ }^{3,9}$
}

To cite: Barman M, Murray F, Bernardi Al, et al. Nutritional impact on Immunological maturation during Childhood in relation to the Environment (NICE): a prospective birth cohort in northern Sweden. BMJ Open 2018;8:e022013. doi:10.1136/ bmjopen-2018-022013

\section{- Prepublication history} and additional material for this paper are available online. To view these files, please visit the journal online (http://dx.doi.org/ 10.1136/ bmjopen-2018-022013).

Received 29 January 2018 Revised 10 July 2018 Accepted 20 August 2018
Check for updates

(C) Author(s) (or their employer(s)) 2018. Re-use permitted under CC BY-NC. No commercial re-use. See rights and permissions. Published by BMJ.

For numbered affiliations see end of article.

Correspondence to

Dr Malin Barman;

malin.barman@chalmers.se

\section{ABSTRACT}

Introduction Prenatal and neonatal environmental factors, such as nutrition, microbes and toxicants, may affect health throughout life. Many diseases, such as allergy and impaired child development, may be programmed already in utero or during early infancy. Birth cohorts are important tools to study associations between early life exposure and disease risk. Here, we describe the study protocol of the prospective birth cohort, 'Nutritional impact on Immunological maturation during Childhood in relation to the Environment' (NICE). The primary aim of the NICE cohort is to clarify the effect of key environmental exposures-diet, microbes and environmental toxicants-during pregnancy and early childhood, on the maturation of the infant's immune system, including initiation of sensitisation and allergy as well as some secondary outcomes: infant growth, obesity, neurological development and oral health.

Methods and analysis The NICE cohort will recruit about 650 families during mid-pregnancy. The principal inclusion criterion will be planned birth at the Sunderby Hospital in the north of Sweden, during 2015-2018. Questionnaires data and biological samples will be collected at 10 timepoints, from pregnancy until the children reach 4 years of age. Samples will be collected primarily from mothers and children, and from fathers. Biological samples include blood, urine, placenta, breast milk, meconium, faeces, saliva and hair. Information regarding allergic heredity, diet, socioeconomic status, lifestyle including smoking, siblings, pet ownership, etc will be collected using questionnaires. Sensitisation to common allergens will be assessed by skin prick testing and allergic disease will be diagnosed by a paediatrician at 1 and 4 years of age. At 4 years of age, the children will also be examined regarding growth, neurobehavioural and neurophysiological status and oral health.

Ethics and dissemination The NICE cohort has been approved by the Regional Ethical Review Board in Umeå, Sweden (2013/18-31M). Results will be disseminated through peer-reviewed journals and communicated on scientific conferences.

\section{Strengths and limitations of this study}

- Prospective study design covering a period from mid-pregnancy to the age of 4 years with biological samples from both the mother, father and child for evaluation of microbiology, nutrition, immunology, environmental toxicants, genetics and epigenetics.

- Interdisciplinary, translational approach and advanced analytical methods within the field of allergology, immunology, nutrition, microbiology, toxicology, obstetrics, childhood growth, neurophysiological development and oral health.

- The primary outcome, allergic disease (food allergy, atopic eczema, asthma and allergic rhinoconjunctivitis) is diagnosed by a paediatric allergology specialist according to strictly defined protocols.

- The large participant burden may also result in selection bias and loss to follow-up.

- Although the study sample will allow in-depth analyses of the effect of multiple exposures and genetic factors on several outcomes, these analyses may largely be exploratory and may require confirmation in follow up-studies.

\section{INTRODUCTION}

The fetal and neonatal periods could be regarded as a window of opportunity for influencing health during childhood and throughout life. Environmental exposures, such as nutrition, microbiota and toxicants and genetic or epigenetic factors may synergise to impact growth, neurodevelopment and immune maturation. However, which factors are most important and the mechanisms by which they operate are still poorly understood. The Nutritional impact on Immunological maturation during Childhood in relation to the Environment (NICE) cohort focuses primarily on early life 
exposures, immune maturation and risk of immune-mediated diseases.

Allergy, dental caries and overweight are the most common chronic conditions in childhood, affecting the health and well-being of around 25\%,20\% and 13\% of children, respectively. ${ }^{1}$ Chronic diseases place a substantial pressure on both the individual and on the healthcare system. Hence, it is important to explore how lifestyle, environmental and genetic/epigenetic factors during pregnancy and the first years of life protect against or facilitate the development of chronic diseases.

Allergy is characterised by dysregulation of the immune system leading to an incapacity to develop tolerance to normally harmless proteins in food and air. Decreased exposure to microorganisms and reduced complexity of the gut microbiota in early life have been associated with an increased risk for the development of allergy. ${ }^{23}$ Also, the diet of pregnant and lactating women ${ }^{4}$ and their exposure to environmental toxicants ${ }^{56}$ have been suggested to play a role in immune maturation and allergy development. In spite of extensive research in this area, the specific causes and pathways leading to immune maturation, and their subsequent protective or detrimental influences on allergy development, remain largely unknown.

In order to identify the key factors leading to the development of healthy immune regulation or allergic disease, it is necessary to study immune events at a very early age, ${ }^{7}$ and account for external factors that could influence immune development. Allergy may present as early as a few months of age, usually manifested as atopic eczema or food allergy, while asthma and rhinoconjunctivitis generally appear at school age. A major source of imprecision in many studies on allergy development is the diagnostic methodology. While questionnaires are convenient and cost-effective, the diagnosis of childhood allergy by a skilled clinician, aided by tests of sensitisation, is the gold standard. For food allergy, a careful investigation of medical history by an experienced clinician, aided by elimination-provocation is the only method to adequately identify this type of allergic manifestation.

Multiple environmental factors, including diet and exposure to toxicants, may affect both the immune system and neurophysiological development. ${ }^{8-11}$ Nutritional factors considered to be immunomodulatory include unsaturated fatty acids, vitamins $\mathrm{A}$ and $\mathrm{D}$, iron and zinc. Some nutrients as well as some metals pass the placenta to the fetus. Some metals accumulate in the placenta, thus elemental metals may cause both impaired placental function and fetal toxicity. Even low-level exposure to certain metals, for example, cadmium (Cd), arsenic (As), manganese $(\mathrm{Mn})$ and lead $(\mathrm{Pb})$, has been found to impair fetal and childhood immune function, growth and neurodevelopment. ${ }^{12-15}$

Overweight and obesity are increasing worldwide. Overweight at a young age is an important risk factor for obesity later in life. In Sweden, obesity has increased during the last 40 years from $2.3 \%$ to $4.7 \%$ among girls and from $2.6 \%$ to $8.5 \%$ among boys. ${ }^{16}$ Socioeconomic status and physical activity are important determinants, but there are other factors to explore such as the impact of different dietary components (eg, fatty acids) and environmental toxicants, metabolic processes and epigenetic factors, to name just a few.

In Western countries, one-fifth of children are so-called high-caries cases (children with a high burden of dental caries) that do not respond to traditional prevention. ${ }^{17}$ These high-caries cases derive from an immune deficiency or carriage of high virulence strains of Streptococcus mutans, consistent with their non-responder behaviour. ${ }^{18}{ }^{19}$ By contrast, the low-to-moderate risk children develop caries as expected from eating and oral hygiene habits. ${ }^{18}$ Therefore, the NICE study aims to evaluate the oral health of all participating children in order to better understand the link between impaired immune maturation and the risk for caries development.

\section{AIMS AND OBJECTIVES}

\section{Primary aim}

The primary aim of the NICE birth cohort is to evaluate how multiple environmental exposures such as lifestyle, diet, microbes and toxicants influence maturation of the immune system and affect allergy development.

\section{Secondary aim}

The secondary aim of NICE is to examine if these same environmental exposures influence pregnancy outcomes, childhood growth, obesity, neuropsychological development and caries development.

\section{Research questions}

The NICE cohort has been designed to answer the following research questions:

i. Which nutrients affect the maturation of the infant's immune system, allergy development, oral health, neurophysiological development, growth and obesity?

ii. Can a metabolic fingerprint be identified that predicts disease risk during childhood?

iii. What role do the oral and gut microbiota play in immunological maturation, allergy development, oral health, neurophysiological development, growth and obesity?

iv. What is the impact of early life exposure to toxicants, particularly toxic metals, on immune maturation, allergy development, caries diseases, neurophysiological development, growth and obesity?

v. Does maturation of the immune system during fetal life and infancy affect the risk of allergy development, oral health or neurophysiological development?

vi. Can certain immune signatures be identified that are associated with immune dysregulation, caries development or, conversely, with health?

vii. Is early life exposure to toxic metals and essential elements reflected in the dental enamel? 
viii. Which nutritional, environmental and lifestyle profiles are protective against caries in the high versus the low-to-moderate caries risk groups and types?

ix. What is the impact of nutrition and toxic exposures on the epigenetic programming of children in relation to immune maturation, allergy development, oral health, neurophysiological development, growth and obesity?

x. What are the effects of perinatal exposures (diet, lifestyle and environmental toxicants) on pregnancy outcomes such as gestational diabetes, pre-eclampsia, pregnancy length, intrauterine growth and birth weight?

\section{METHODS AND ANALYSIS \\ Study design and setting}

The study design is a prospective longitudinal birth cohort study. All expectant parents planning to give birth at the Sunderby Hospital in Luleå, during 2015-2018, will be invited to participate. The Sunderby Hospital, with approximately 2000 deliveries per year, is located in Region Norrbotten in the very north of Sweden. Region Norrbotten makes up almost a quarter of Sweden geographically, but has a population of only approximately 250000 inhabitants. Expectant mothers are cared for at 22 maternity clinics across the region. Routine ultrasound is performed at gestational week 18-19 at either of three sites: at the Sunderby, Kalix or Piteå hospitals. These three hospitals have combined catchment area of approximately 200000 inhabitants.

\section{Recruitment and inclusion and exclusion criteria}

All expectant parents living in the catchment area of the Sunderby Hospital will receive a concise information leaflet about the study at their first visit to their local maternity clinic in gestation week 10-12. Recruitment will take place in connection with the routine ultrasound at gestational week 18-19. All expectant parents will be given detailed information about the study. The parents who wish to participate will be requested to provide informed consent. They will subsequently receive an acknowledgement letter and will be assigned a study ID number.

The criteria for participation in the study will be as follows: the intention to give birth at the Sunderby Hospital, the ability to communicate in written and spoken Swedish and current habitation in Region Norrbotten. Families will be excluded from the study if they move from Norrbotten before the child is born. Families who move outside of Norrbotten after birth will be allowed to continue participation in the study if so wished and follow-up appointments will be arranged where possible.

\section{Estimated sample size}

The NICE cohort will be based on the conservative inclusion of children to be delivered at the Sunderby Hospital with run-in during the period 15 January 2015 to 28 February 2018 (table 1 ). The aim is to include 650 families. Assuming 25\% allergy rate among 650 families, we estimate the effects that can be detected with $80 \%$ power and 5\% significance level in univariate analysis of marginal effects. For continuous variables, an effect size (Cohen's d) of 0.25 can be detected and for dichotomous exposures ranging in prevalence among allergic patients $(5 \%, 10 \%, 20 \%, 50 \%)$, the corresponding ORs that can be detected are 5.3, 2.9, 2.0 and 1.7, respectively.

\section{Study outcomes}

\section{Primary outcome}

The primary outcome in NICE is immune maturation in the neonatal period and its effect on development of sensitisation and allergy over a follow-up period of 4 years.

\section{Secondary outcomes}

The secondary outcomes are:

Pregnancy outcomes (pre-eclampsia, gestational diabetes, gestational week at birth, intrauterine growth and birth weight).

Child growth (including overweight and obesity).

Neurophysiological development (test of motor, cognitive and behavioural function).

Caries and oral health.

\section{Biological sample collection}

Mothers, fathers and infants participating in the NICE cohort will be repeatedly followed up from pregnancy until 4 years of age with both questionnaires and biological sample collection (figure 1). Mothers will fill out questionnaires and/or give biological samples at gestational week 18, 28 and 34, at delivery and at 1, 4 and 12 months post partum. Fathers will fill out questionnaires

Table 1 Overview of the NICE study recruitment and follow-up

\begin{tabular}{l|l|l|l|l|l|l|l|l|l}
\hline \multicolumn{2}{l}{2014} & 2015 & 2016 & 2017 & 2018 & 2019 & 2020 & 2021 & 2022 \\
\hline Project start, planning & & & & & & & & & \\
\hline Recruitment of patients & & & & & & & & & \\
\hline Children born & & & & & & & & & \\
\hline 12 months follow-up & & & & & & & & & \\
\hline 48 months follow-up & & & & & & & & & \\
\hline Data collection & & & & & & & & & \\
\hline
\end{tabular}

NICE, Nutritional impact on Immunological maturation during Childhood in relation to the Environment. 


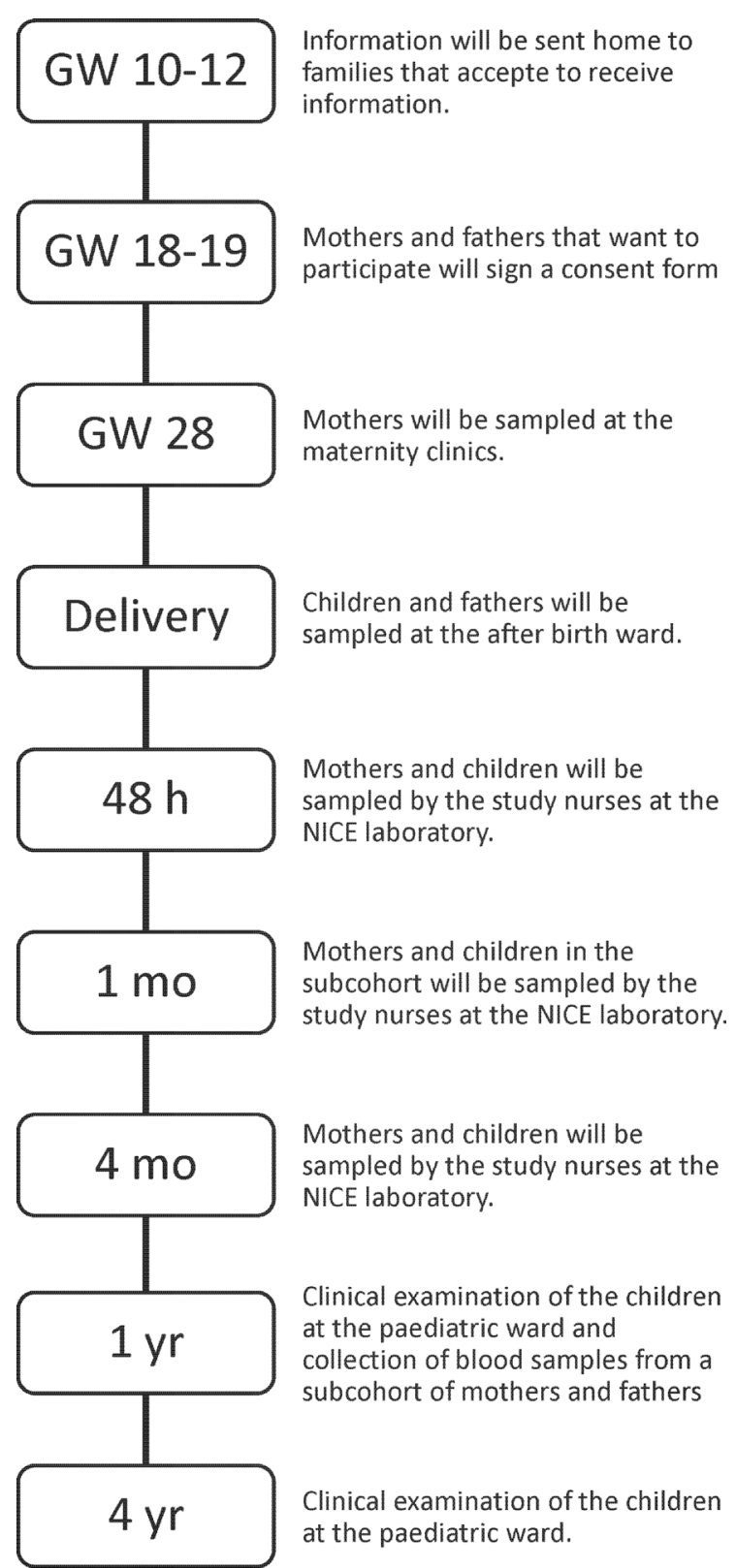

Figure 1 Flow chart of the NICE cohort. GW, gestational week; NICE, Nutritional impact on Immunological maturation during Childhood in relation to the Environment.

and/or give biological samples at gestational week 18 and 48 hours and 12 months post partum. All children will be followed up with biological samples or clinical examinations at birth and at 48 hours, $4,12,18$ and 48 months of age. The flow chart of the study is shown in figure 1 and a detailed overview of the collection of biological samples and questionnaires is shown in table 2.

\section{Subcohort}

Approximately one in four recruited families will be offered the possibility to participate in a subcohort with more extensive sampling of mother and infant (table 2). Mothers and children in the subcohort will be followed up additionally at 1 month of age. Also, children in the subcohort will collect faeces samples at weeks 1, 2, 4 and
6 and at months 4 and 6 . The assignment of families to the subcohort will be performed at delivery, based on the feasibility of extended sampling at that timepoint.

\section{Blood samples}

Blood samples collected from the parents will include: $10 \mathrm{~mL} / 5 \mathrm{~mL}$ SST II Advance serum tube, $10 \mathrm{~mL} / 3 \mathrm{~mL}$ EDTA tube (all from Becton Dickinson, Plymouth, UK), $6 \mathrm{~mL}$ Na-heparin trace element-free tube (Greiner bio-one, Kremsmünster, Austria) and $10 \mathrm{~mL}$ RNA-stabilised blood collection Tempus tubes (Applied Biosystems, Foster City, California, USA), the latter from mothers only. The mothers will be asked to be fasting for 8 hours before study visits.

At birth, venous and arterial cord blood will be collected from the umbilical cord into small $500 \mu \mathrm{L}$ Microtainer EDTA tubes (Becton Dickinson) using a syringe. Mixed umbilical cord blood will be collected into another 3 and $6 \mathrm{~mL}$ EDTA tubes (the $3 \mathrm{~mL}$ tube will be stored at room temperature for subsequent flow cytometric analysis) and a $6 \mathrm{~mL}$ Na-heparin trace element-free tube (Greiner bio-one). Midwives will follow WHO guidelines with regard to umbilical cord clamping, that is, cord clamping will be carried out $>1$ min after the birth or when cord pulsation has ceased.

Venous blood will be collected from the infants by the study nurses into $3 \mathrm{~mL}$ EDTA tubes (Becton Dickinson) using a Microneedle (Sarstedt, Nümbrecht, Germany) or Eclipse Signal Blood Collection needle (Becton Dickinson). Blood will be drawn from the dorsal side of the hand at 48 hours, 1 month, 4 months and from the hand or arm at 12 and 48 months of age.

\section{Urine samples}

Infant's urine at 4 months of age will be collected in a urine collection bag placed around the penis or vagina under the infant's nappy at the start of a study visit, with the bag being collected at the end of the study visit. Urine samples at 4 years of age will be collected in a potty lined with a plastic bag. Midstream urine samples, taken at a random timepoint during the day, will be collected from adults.

\section{Placenta}

After delivery, the placenta will be examined according to local guidelines by the delivery personnel and placed in a plastic bag and stored at $4^{\circ} \mathrm{C}$. The placenta will be collected by the study nurses and processed at the laboratory as outlined in figure 2.

\section{Breast milk}

Mothers will be asked to express $30 \mathrm{~mL}$ of breast milk in the morning before the second breastfeeding occasion of the day, at 1 and 4 months post partum. Samples will be collected in a clean bowl or pump and poured into plastic centrifuge tubes for immediate storage at $-20^{\circ} \mathrm{C}$ at home. Mothers will be requested to bring the frozen tubes with them at the 4 months follow-up and reminded to keep the samples in a cooling bag or wrapped in paper with a 


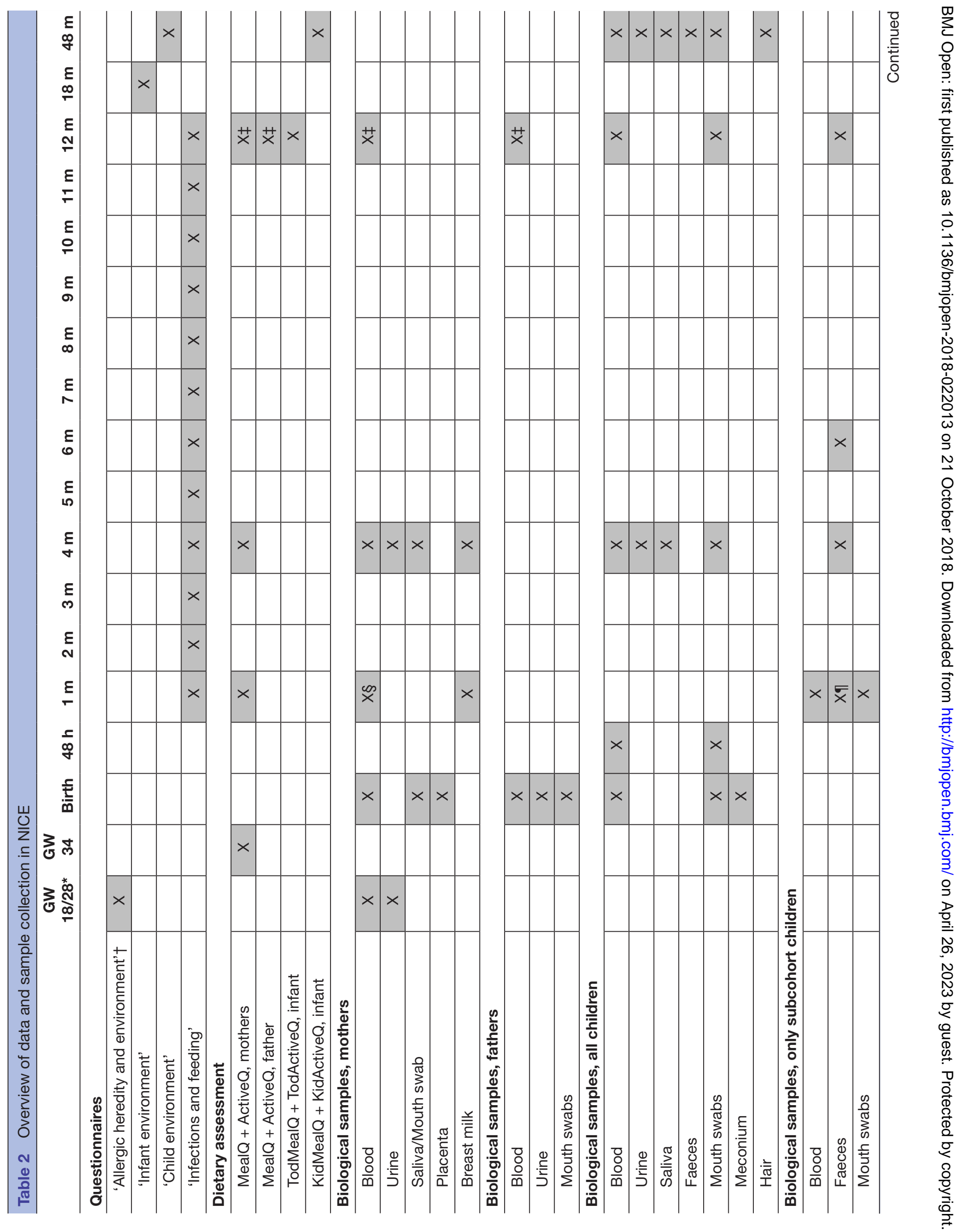




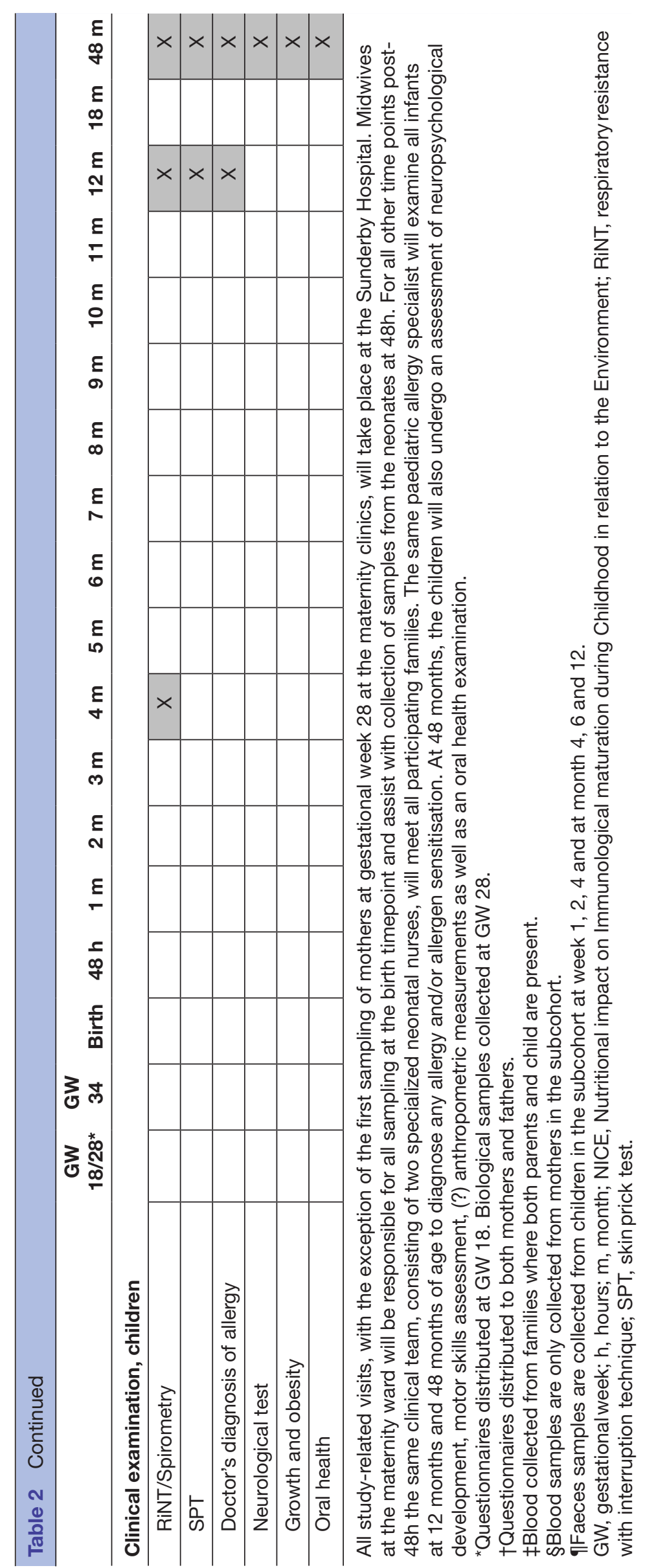


PLACENTA

(Basal plate)

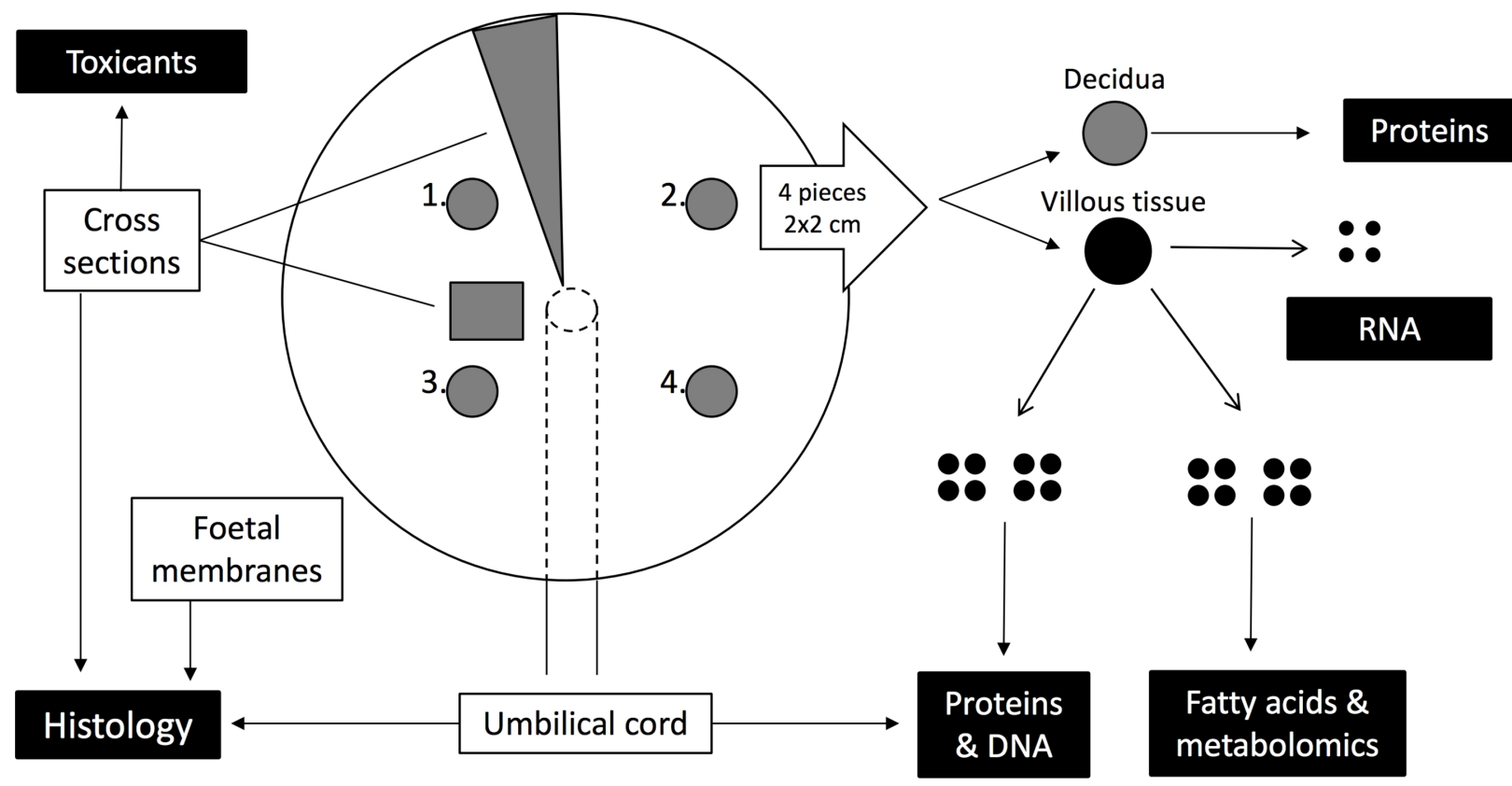

Figure 2 Schematic overview of placenta collection at delivery. Samples from the placenta will be collected for analysis of histology, DNA, RNA, proteins, metabolomics, fatty acids, toxicants and essential elements. First four pieces, $4 \mathrm{~cm}^{3}$ of villous tissue including decidua will be collected from the basal plate from four separate places of the placenta. The decidua will be removed stored in a separate cryo-tube. The remaining villous tissue will be divided into 20 smaller pieces, 4 pieces of $3 \mathrm{~mm}$ $\varnothing$ that will be put in RNAlater and 16 pieces that will be put 4 and 4 in separate cryo-tubes. Two different cross-sections will be then collected: one cross-section measuring approximately $2 \times 2 \mathrm{~cm}$ taken within $4 \mathrm{~cm}$ from the cord insert will be placed in formalin. Another cross-section formed as a V taken from the cord insert to the end of the placenta will be sampled with specific metal-free ceramic knife and put into plastic bags. The cross-sections will be taken with care to avoid selecting a tissue section that has previously been sampled for villous tissues. At $1 \mathrm{~cm}$ from the cord insert, a $2 \mathrm{~cm}$ piece of the umbilical cord will be sampled and stored in formalin. From the same end of the cord, two pieces, $1 \mathrm{~cm}$ each in length, will be sampled and placed in a cryo-tube. Also, samples from the membrane will be collected: pieces of the amnion and chorion membranes will be placed in separate cryo-tubes, while one piece of membrane with both the membranes still attached to each other ('double membrane') will be placed in formalin.

cooling block to prevent thawing of the samples during transport to the hospital. The samples will be transferred directly to a $-80^{\circ} \mathrm{C}$ freezer on arrival to the research laboratory. If thawing appears to have occurred during transport, this will be noted in the sample preparation protocol.

\section{Meconium}

Meconium will be sampled when voided using a sterile spatula and put in a sterile container that will be immediately stored at $4^{\circ} \mathrm{C}$ until aliquoting at the research laboratory.

\section{Faeces}

Faecal samples will be collected from the infant's nappy by the parents at home using a sterile spatula. Faeces will be put in sterile tubes prelabelled with the child's study ID. Parents will be requested to write the date of sampling on the tubes and then place the samples in the freezer as soon as possible and to bring it to the hospital at the next follow-up. Participants will be requested to keep the samples in a cooling bag or wrapped in newspaper to prevent thawing of the samples during transport to the hospital. The stool samples will be transferred directly to a $-80^{\circ} \mathrm{C}$ freezer on arrival to the research laboratory. If thawing appears to have occurred during transport, this will be noted in the sample preparation protocol.

\section{Saliva}

Approximately $1.5-2 \mathrm{~mL}$ saliva will be collected from mothers, by spitting into a plastic tube. Infant saliva (approximately $0.5 \mathrm{~mL}$ ) will be collected from the buccal cavity using a plastic Pasteur pipette.

\section{Buccal swabs}

Buccal swabs (eSwab, Copan, Bresia, Italy) will be collected by swiping the swab tip pass beneath the upper lip, and the inside of the cheeks, taking care to avoid touching the teeth. The swabs will be stored at $4^{\circ} \mathrm{C}$ until centrifugation at $12000 \mathrm{~g}$ for $10 \mathrm{~min}$. Excess fluid will be removed from the bacterial pellet before storage.

\section{Hair specimens}

At 48 months, hair specimens will be cut by stainless steel scissors from the occipital part of the head, beneath some covering hair, as close to the scalp as possible. 


\section{Handling, transport and storage of biological samples}

At gestational week 28, samples will be collected at the maternity clinics. The RNA Tempus blood samples and urine samples will be stored immediately at $4^{\circ} \mathrm{C}$, while blood samples will be kept at room temperature for $30 \mathrm{~min}$ before centrifugation. All samples will be stored at $4^{\circ} \mathrm{C}$ until transport and will be subsequently transported at $4^{\circ} \mathrm{C}-10^{\circ} \mathrm{C}$ to the hospital laboratory, either the same day or the following work day.

Samples taken at delivery and at 48 hours will be stored at $4^{\circ} \mathrm{C}$ and processed within 2-12 hours if sampled on weekdays and within 12-65 hours if sampled on weekends. Samples taken at the follow-up visits at 1, 4, 12 and 48 months of age will be collected by the NICE cohort study nurses and handled the same work day. All biological samples will stand for 15-30 min in room temperature until centrifugation of the blood. After centrifugation of the blood, all samples will be stored at $4^{\circ} \mathrm{C}$ until aliquoting (performed on a cooling block) and interim storage at $-20^{\circ} \mathrm{C}$ (for a maximum of 12 weeks). Once filled, storage boxes will be transferred to long-term storage at $-80^{\circ} \mathrm{C}$. Hair samples will be collected in paper envelopes and stored at room temperature. A laboratory protocol will be filled in for all samples where all transport and preparation times will be noted in order to be able to account for the time from sampling to freezer in all downstream analyses.

\section{Collection of participant data}

All study participants will be closely followed up during pregnancy from inclusion until the delivery from physician's notes in their medical records. Data from the medical pregnancy records will be analysed and collected in the database.

At inclusion, both parents will be asked to fill out one online questionnaire each regarding allergic heredity and environment. When the infant has reached 18 months of age, the second online questionnaire, the 'Infant environment' questionnaire, regarding the infant's current living environment and lifestyle will be sent. The third online questionnaire, the 'Child environment' questionnaire will be sent out to the participants when the infant
Table 4 Overview of the dietary assessments and activity questionnaires in the NICE-cohort

\begin{tabular}{|c|c|c|}
\hline Questionnaire & Time & Answered by \\
\hline Meal-Q1+Active-Q1 & GW 34 & Mothers \\
\hline Meal-Q2+Active-Q2 & 1 month & Mothers \\
\hline Meal-Q3+Active-Q3 & 4 months & Mothers \\
\hline TodMeal-Q+TodActive-Q & 12 months & $\begin{array}{l}\text { Parents answer } \\
\text { for the infant }\end{array}$ \\
\hline KidMeal-Q+KidActive-Q & 48 months & $\begin{array}{l}\text { Parents answer } \\
\text { for the infant }\end{array}$ \\
\hline
\end{tabular}

GW, gestational week; NICE, Nutritional impact on Immunological maturation during Childhood in relation to the Environment.

has reached 48 months of age and concerns the infant's environment and lifestyle (tables 2 and 3 ).

\section{Assessment of dietary intake in mothers and children}

The mothers will receive a web-based, food frequency questionnaire (Meal-Q) by email during pregnancy in gestational week 34 and at 1 and 4 months post partum, consisting of questions regarding their diet during the previous month ${ }^{20}$ (tables 2 and 4). Reminders will be sent out by email 1 and 2 weeks after the initial email and, alongside the last reminder, a text message will be sent to their cell phones. The questionnaires will have a meal-based and interactive format, that is, only those who report a relevant frequency of consumption of a certain food or food group will receive follow-up questions, to promote ease of use and minimise the time required to answer the questionnaire. Depending on the number of follow-up questions, the Meal-Q questionnaire contains 102-174 questions.

When the child reaches 12 and 48 months of age, the parents fill out a food frequency questionnaire about the infant's food habits (TodMeal-Q and KidMeal-Q, respectively), which will be similar to the Meal-Q but adopted for young children. ${ }^{22}$ Parents from families where both parents have attended the follow-up visits will be asked to participate in a subcohort with extended sampling,

Table 3 Overview of questionnaires distributed in the NICE cohort

\begin{tabular}{llll}
\hline Questionnaire & Time & Answered by & Questions about \\
\hline $\begin{array}{l}\text { Q1: 'Allergic } \\
\text { heredity and } \\
\text { environment' }\end{array}$ & GW 20-25 & $\begin{array}{l}\text { Mothers and } \\
\text { partners answer one } \\
\text { questionnaire each }\end{array}$ & $\begin{array}{l}\text { Education, profession, nationality, allergic heredity, smoking habits, } \\
\text { alcohol habits, residence, heating, ventilation, 'mould', siblings, pets. }\end{array}$ \\
$\begin{array}{l}\text { Q2: 'Infant } \\
\text { environment' }\end{array}$ & 18 months & $\begin{array}{l}\text { Parents answers } \\
\text { about the family }\end{array}$ & $\begin{array}{l}\text { Questions to both parents regarding: profession, education, ethnicity, } \\
\text { civil status, smoking habits, hunting habits, residence. } \\
\text { Questions about the infant: feeding, vaccinations, travels, daycare, } \\
\text { behaviour, siblings, pets. }\end{array}$ \\
$\begin{array}{l}\text { Q3: 'Child } \\
\text { environment' }\end{array}$ & 48 months & $\begin{array}{l}\text { Parents answers } \\
\text { about the family }\end{array}$ & $\begin{array}{l}\text { Questions to both parents regarding: profession, education, civil } \\
\text { status, smoking habits, hunting habits, residence } \\
\text { Questions about the infant: feeding, vaccinations, travels, daycare, } \\
\text { behaviour, siblings, pets. }\end{array}$ \\
\hline
\end{tabular}

GW, gestational week; NICE, Nutritional impact on Immunological maturation during Childhood in relation to the Environment. 
including a Meal-Q questionnaire for each of the parents at 12 months of age of the child.

To measure total energy expenditure, all dietary questionnaires will be combined with the activity questionnaires Active- $\mathrm{Q}^{23}$ or KidActive- $\mathrm{Q}^{24}$

The questionnaires Meal-Q and KidMeal-Q have been validated in their original form, ${ }^{20-22}$ but all dietary questionnaires were modified to better fit the specific needs of the study of evaluation of fat quality, consumption of fish from polluted areas, sugar and probiotic use. Modifications are detailed in online supplementary file 1 . Nutritional composition of the diet will be calculated from the dietary questionnaires using the database of nutrient content published by the Swedish National Food Agency. The nutrient conversion for the questionnaires will be calculated by computer programs developed specifically for those questionnaires.

\section{Diary for recoding introduction of complementary foods to the infant diet}

Practices of introduction of complementary foods and formulas to the infant's diet will be assessed each month during the first 12 months of life by a web-based questionnaire; 'Infections and feeding' (table 2). The questions include the infant's intake of breast milk from breast or bottle, and whether certain foods have been introduced, including formula (percentages of total feeding and brand name), gruel (regular, with/without gluten or lactose, soya based), porridge (regular, without gluten or lactose) and different food groups (potatoes/roots, fruit/ juice, berries, nuts/almonds, peanuts/peanut oil, bread/ crackers/cookies, butter, margarine, oil, milk, ice-cream, sour milk, yoghurt, meat, fish and egg), as well as if there are any food the child has started to eat more than twice per week that is not included above. Additional questions will be asked about probiotics, vitamin $\mathrm{D}$, omega-3 or other supplements and frequency of canned versus home-made food.

Parents will be asked to estimate the proportion of commercial versus home-made baby food. In order to be able to adjust the statistical analyses for reverse causation, the parents will be also asked the questions: 'during the past month, have you actively avoided to give any type of food due to concern of allergy?' and 'if so what food items have been excluded from the child's diet?'

\section{Outcome measures}

Infections during year 1

Infectious episodes will be recorded monthly by the parents during the first 12 months using the above-mentioned web-based questionnaire. If an infectious episode is recorded, follow-up questions will be posed concerning duration of the illness, body temperature, breathing problems, antibiotic treatment, medications, hospitalisation, etc.

\section{Lung function test}

Lung function will be assessed at 4 and 12 months, using respiratory resistance with interrupter technique (MicroRint, MicroMedical, UK). The method estimates alveolar pressure (Palv) by performing a rapid occlusion of the airways during the respiratory circle at rest, assuming that Palv will equal mouth pressure (Pmo). Respiratory resistance with interrupter technique (RInt) is a non-invasive method that requires minimal cooperation and can be used in evaluating airway obstruction in uncooperative and small children. ${ }^{25} 26$ At 48 months, lung function will be measured by standardised Impulse Oscillometry.

\section{Skin prick test}

Sensitisation to common allergens will be assessed by skin prick testing at 12 and 48 months of age. Testing will be performed on the volar side of the lower arm and will be carried out according to the standards of the Subcommittee on Skin Tests of the European Academy of Allergy and Clinical Immunology. ${ }^{27}$ Six different standardised allergens (egg, milk, birch, timothy, cat and dog) will be applied with a lancet; histamine dihydrochloride (10 mg/ $\mathrm{mL}$ ) will be used as positive control (Soluoprick, ALK Nordic A/S, Denmark). The test will be considered positive for a particular allergen if the mean weal diameter is at least $3 \mathrm{~mm}$ after $15 \mathrm{~min}$.

\section{Allergy diagnosis}

Allergy will be diagnosed at 12 and 48 months by a specialist in paediatric allergology. The diagnosis will be based on a clinical history and a clinical examination using the same methodology as in the BAS cohort, ${ }^{28}$ enabling direct comparisons. The clinical protocol will be carefully standardised and complies with the diagnostic protocols of our previous ALLERGYFLORA study ${ }^{29}$ and FARMFLORA study ${ }^{4}$.

The following diagnostic criteria will be used for clinical allergic diagnosis at 12 and 48 months of age.

\section{Atopic eczema, atopic dermatitis}

Various diagnostic criteria for atopic dermatitis (AD) have been proposed and validated. For the NICE cohort follow-up at 12 and 48 months, we choose to employ the simplified criteria proposed by Williams $e t$ al with high sensitivity and specificity. ${ }^{30-32}$

- Major criteria (=must have): an itchy condition or parental report of scratching/rubbing by the child.

- Plus three or more of the following criteria: 1) personal history of itchiness in skin creases such as cheeks, folds of the elbows, behind knees, front of ankles or around the neck; 2) personal history of asthma or hay-fever or other atopic disease or a history of atopic disease among a first-degree relative; 3) a personal history of generally dry skin in the previous year; 4) visible eczema on the cheeks, folds of the elbows, behind knees, front of ankles or around neck; 5) a debut before 24 months of age. 


\section{Food allergy}

A diagnosis of food allergy requires a medical history of allergic reactions, on at least two consecutive occasions when eating a specific food allergen. The allergic reaction disappears on elimination of the allergen. For early food reaction, allergic symptoms will appear $<2$ hours with rush, itching, urticaria, angioedema, gastrointestinal pain, vomiting, oral symptoms, asthma or anaphylactic reaction. Late food reaction ( $>2$ hours) will present eczema, gastrointestinal reactions with pain, diarrhoea, obstipation and weight loss. For all suspected cases of food allergy, the diagnosis will be confirmed by elimination-provocation, that is, the child will be given the suspected food allergen and symptoms will be recorded. The elimination-provocation procedure will be carried out at home following local guidelines if the symptoms are mild. If this is not feasible, or if the case is unclear, the child will be admitted to the paediatric ward at the Sunderby Hospital for repeated open food provocation until the diagnosis is clarified (food allergic or healthy). Open provocation will start with a dose determined by the individual child's food allergen reaction and will be followed by increasing doses of specific food allergen given with $30 \mathrm{~min}$ interval in three to five steps. The child will stay at the ward for 2 hours after the last dose and parents will thereafter report any symptoms during the following days.

\section{Allergy to animals}

A diagnosis of allergy to animal dander requires a medical history of symptoms from the eyes and/or nose and/or asthma on at least two consecutive occasions when exposed to a specific animal dander (eg, dog, cat, rabbit, horse). The reaction disappears when the animal allergen is removed.

\section{Pollen allergy}

A diagnosis of pollen allergy requires a medical history of symptoms from eyes and/or nose and/or asthma on two consecutive occasions when exposed during pollen season. The reaction disappears in areas with lower pollen content or following medication.

\section{Asthma}

At 12 months, an asthma diagnosis requires at least one of the following: 1) the presence of wheeze between infections, 2) persistent wheeze for at least 4 weeks, 3) at least one period of wheeze during an infectious disease with concomitant allergic disease ( $\mathrm{AD}$, food allergy or allergic rhinoconjunctivitis) or 4) three episodes of wheeze during infectious disease, with no concomitant allergic disease.

At 48 months, an asthma diagnosis requires at least one of the following: the presence of 1) wheeze within the last 12 months (during or between infections) or 2) ongoing controller medication.

If there is any doubt concerning the asthma diagnosis at 12 or 48 months, the child will be admitted to the children's clinic at the Sunderby Hospital for a second clinical follow-up for doctor's diagnosed asthma.

\section{Neuropsychological and neurobehavioural examination}

At 48 months of age, the parents will be asked to answer questionnaires and the child will undergo examination with focus on neuropsychological behaviour. The questionnaires will consist of parent-reported Child Behaviour Checklist, Adaptive Behaviour Assessment System) and Social Responsiveness Scale. Cognitive development will be tested face-to-face with Wechsler Preschool and Primary Scale of Intelligence and a physical assessment of motor skills with Peabody Development Motor Scales (by a physiotherapist).

\section{Oral health examination}

Caries will be recorded in children at 48 months of age by one to three experienced and calibrated dentists (intra-examiner and interexaminer kappa $\geq 0.98$ ) using a mirror and probe as the number of decayed (enamel caries included), filled surfaces in the primary dentition (defs). ${ }^{17-19}$ At the same visit, caries will also be recorded for the parents using two bitewing radiographs as the number of decayed (enamel caries included), filled surfaces in the permanent dentition (DeFS). Whole saliva and buccal swabs will be collected from the parents. Complementary questionnaires on oral behaviour data, such as oral hygiene, intake frequency of sweets and sugary drinks, use of fluorides and oral hygiene, will be taken for children and parents. Enamel discolourations and irregularities on visual inspection and probing will be registered and exfoliated primary teeth will be collected by parents in prepared tubes.

\section{Analyses}

\section{Immune analysis}

Blood samples will be analysed by flow cytometry regarding numbers and percentages of $\mathrm{T}$ and $\mathrm{B}$ cells and their subsets and their markers of naivety, activation and memory. ${ }^{33} \mathrm{~A}$ saliva sample will be collected from the mother close to the time of birth and from the infant at 4 months for the analysis of secretory IgA and salivary microbiota. ${ }^{35}$

\section{Genetic analyses}

Genomic DNA will be extracted from blood collected in EDTA tubes from mothers, fathers and infants for analysis of genetic variation. Blood will also be collected from the mothers in Tempus Blood RNA Tubes for stabilisation and isolation of total RNA from whole blood for gene expression analysis.

\section{Metabolomics}

To study metabolic alterations in the participants, non-targeted metabolomics profiling will be employed on plasma samples by liquid chromatography mass spectrometry (LC-MS) and gas chromatography mass spectrometry (GC-MS) analysis. ${ }^{36}$ 


\section{Transcriptomics}

Whole transcriptome analysis will be performed on placenta tissue from those placentas sampled within 4 hours from birth and on maternal blood collected in Tempus RNA stabiliser tubes at gestation, at birth and during lactation.

Total RNA will be analysed and its quality will be checked using a bioanalyser. RNA microarray analysis will be performed by next-generation sequencing and the raw data will be analysed using $\mathrm{R}$ biostatistical computing platform RStudio GUI. Gene set enrichment analysis will be performed to visualise regulated biological processes. ${ }^{37}$

\section{Epigenetics}

Whole-epigenome gene-specific methylation analysis (Illumina EPIC) will be performed on selected samples of the placenta, cord blood and blood of children at 48 months of age to identify persisting gene-specific changes in DNA methylation. Significant DNA methylation markers will be validated by pyrosequencing in all children. ${ }^{38} 39$

\section{Fatty acid and vitamin A and D composition in blood cell}

\section{membranes and plasma}

Blood cell fatty acid composition will be analysed in unwashed red and white blood cells. Fatty acids from blood cells will be extracted ${ }^{40}$ and analysed using gas chromatography-flame ionisation (GC-FID) ${ }^{41}$ Concentrations of vitamin $\mathrm{A}$ and $\mathrm{D}$ will be determined in plasma with liquid chromathography-tandem massspectometry (LC-MS/MS). ${ }^{42} 43$

Determination of toxic metals, other elements and micronutrients Concentration of toxic and essential elements will be measured in serum $(\mathrm{Ca}, \mathrm{Mg}, \mathrm{Zn}, \mathrm{Se})$, erythrocytes $(\mathrm{Cd}$,
$\mathrm{Mn}, \mathrm{MeHg}, \mathrm{Pb}$ ) and urine (As, $\mathrm{Cd}, \mathrm{U}$ and I) using inductively coupled plasma mass spectrometry (ICP-MS), developed for high-throughput multiple element analyses. ${ }^{44-46}$ Speciation of urinary As (metabolites of inorganic arsenic) will be performed by high-performance liquid chromatography ICP-MS (HPLC-ICP-MS). Urinary F will be measured using an ion-selective electrode.

\section{Oral and gut microbiota analyses}

Infant faeces, oral swab and saliva will be collected for later analysis of microbiota composition using DNA-based sequencing methods. ${ }^{47}$

\section{Data analysis}

Most of the questionnaires will be web-based and data will be downloaded directly from the surveys to SPSS files. All data will be cleaned and entered into a secure database stored within the hospital domain and handled and protected with the same confidentiality as patient journal data. A statistician at the Unit for Research and Innovation, Department of Development, Region Norrbotten, will be responsible for database management. Requests from study researchers for analysis of cohort data require detailed analysis plan is approved by the steering committee. All data transferred to the universities will be blinded so that researchers cannot link individual data to any specific individual.

\section{Statistical analyses}

Univariate and multivariate analysis and data mining, including cluster and hierarchical models, will be used, depending on data and outcome measures. To examine general maternal, paternal and infant demographic data and distribution of outcomes, descriptive analysis will be

Table 5 Overview of research sites involved in the NICE consortium

\begin{tabular}{|c|c|c|}
\hline Institution, Department, Group & $\begin{array}{l}\text { Principal investigator of } \\
\text { the site }\end{array}$ & Main research interests in the NICE cohort \\
\hline Umeå University, Clinical Sciences, Paediatrics & $\begin{array}{l}\text { Anna Sandin, MD, PhD, } \\
\text { Principal Investigator for the } \\
\text { NICE cohort }\end{array}$ & $\begin{array}{l}\text { Environmental exposures in relation to immune } \\
\text { maturation and allergy outcomes. }\end{array}$ \\
\hline $\begin{array}{l}\text { Chalmers University of Technology, Biology } \\
\text { and Biological Engineering, Food and Nutrition } \\
\text { Science }\end{array}$ & $\begin{array}{l}\text { Ann-Sofie Sandberg, } \\
\text { Professor }\end{array}$ & $\begin{array}{l}\text { Nutritional factors in relation to allergy, growth } \\
\text { and neurological function. Metabolomics to } \\
\text { predict risk of disease. Systems biology. }\end{array}$ \\
\hline Gothenburg University, Clinical Microbiology & Agnes Wold, Professor & $\begin{array}{l}\text { Immune maturation, microbiota and allergy } \\
\text { development. }\end{array}$ \\
\hline $\begin{array}{l}\text { Karolinska Institutet, Institute of Environmental } \\
\text { Medicine }\end{array}$ & Marie Vahter, Professor & $\begin{array}{l}\text { Metal exposure and epigenetics in relation to } \\
\text { growth and neurological function. }\end{array}$ \\
\hline $\begin{array}{l}\text { Gothenburg University, Sahlgrenska Academy, } \\
\text { Obstetrics and Gynaecology }\end{array}$ & Bo Jacobsson, Professor & $\begin{array}{l}\text { Pregnancy exposures and outcome with special } \\
\text { interest in placenta. }\end{array}$ \\
\hline $\begin{array}{l}\text { Umeå University, Department of Odontology/ } \\
\text { Cariology }\end{array}$ & $\begin{array}{l}\text { Nicklas Strömberg, } \\
\text { Professor }\end{array}$ & $\begin{array}{l}\text { Nutritional, immune, microbiota and metal } \\
\text { factors in caries development. }\end{array}$ \\
\hline $\begin{array}{l}\text { Karolinska Institutet, Department } \\
\text { of Women's and Children's Health, } \\
\text { Division of Neuropsychiatry, Centre of } \\
\text { Neurodevelopmental Disorders (KIND) }\end{array}$ & Sven Bölte, Professor & $\begin{array}{l}\text { Neuropsychological and neuropsychiatric } \\
\text { assessments. }\end{array}$ \\
\hline
\end{tabular}

NICE, Nutritional impact on Immunological maturation during Childhood in relation to the Environment. 
performed initially. Statistical methods linking outcomes to collected or measured data will depend on the nature of the data: $\mathrm{X}^{2}$ tests will be used for categorical data, Student's t-test and analysis of variance (ANOVA) (and corresponding non-parametric methods when applicable, such as Mann-Whitney U test and Kruskal-Wallis test) for continuous data as well as Pearson's and Spearman's correlations. Questionnaire data will be analysed by factor analysis to investigate interactions between questionnaire variables and identify potential constructs. Similar to principal component analysis (PCA), factors/ constructs can be interpreted as latent variables underlying observed total data variability. To identify independent predictors of outcomes among questionnaire and demographic data (both original data and factor scores), generalised linear mixed models (ANOVA, logistic regressions and conditional logistic regressions) capable of controlling for possible confounders will be applied.

Metabolomics data will be generated and preprocessed at the Chalmers Mass Spectrometry Infrastructure (www. chalmers.se/en/researchinfrastructure/CMSI/Pages/ default.aspx). To identify molecular predictors related to outcomes, metabolomics data will be analysed using predominantly supervised multivariate methods. We will employ partial least squares and random forest techniques incorporated into nested cross-validation frameworks to effectively manage the high-dimensional nature of untargeted metabolomics data. These new techniques also perform automatic, unbiased variable selection, suitable for identifying biomarkers associated with outcome variables. The combined nested cross-validation with unbiased variable selection greatly reduces the risk of false-positive findings while improving modelling accuracy. ${ }^{48}$ Outputs from multivariate models can, as mentioned above, be incorporated into mixed models to be able to adjust for confounders in associations between molecular predictors and outcomes.

Statistical analyses will be performed in two steps: pilot analyses and final analyses. Pilot analyses refer to analysis of a subset of samples collected during 2015-2016, and final analyses refer to data from samples collected from the whole study (2015-2018).

\section{Patient and public involvement}

The study was based on healthy volunteers. Patients and public were neither involved in the design of the study nor in the development of the research questions or outcome measures. Patients and public were not involved in the recruitment or conduction of the study. The results will be disseminated to the study participants on a cohort basis on yearly workshops. No individual results will be given to the participants.

\section{NICE CONSORTIUM}

\section{Steering Committee}

The Steering Committee, consisting of MD, PhD, PI Anna Sandin, Professor Ann-Sofie Sandberg and Professor Agnes Wold, is responsible for administrative and financial coordination and monitoring the progress of the cohort. The Steering Committee will meet twice a year to handle the allocation of resources to the research projects, the recruitment of personnel and the scientific direction.

\section{Research groups}

Research groups involved in the NICE cohort are shown in table 5 .

\section{Collaborators}

Within the NICE cohort, there are close collaborators with a range of expertise. For transcriptomics analysis and systems biology of the NICE samples, we have a close collaboration with Professor Jens Nilsen at Systems Biology at the Department of Biology and Biological Engineering at Chalmers University of Technology in Gothenburg, Sweden. Robert Lundqvist, statistician at the Research and Innovation Unit at Region Norrbotten, will advise on statistical analyses and will be also responsible for the management of the NICE cohort database.

\section{ETHICS AND DISSEMINATION}

The study will be conducted in accordance with the Declaration of Helsinki. The participants will be informed about the aims and the requirements of the NICE cohort, and will be provided both written and verbal information. They will be requested to complete and sign a consent form before being enrolled in the study. Once the infant is born, the parents will again requested to give consent for their child's participation in the study. All participants have the right to withdraw from the study at any point and have their data removed from all study documentation.

All data will be securely stored according to European laws and password protected and accessible by the research team only. All personal data will be blinded so that researchers cannot link individual data to any specific individual. Information resulting from the study will be disseminated to non-scientific audiences in the region where the study has been carried out to keep health professionals and interested members of the public abreast of the findings. Results from the cohort will be disseminated through peer-reviewed journals, preferably open access journals to ensure that all readers from the scientific society, the clinical professionals as well as the general public will have open access to the publication. Results will also be communicated by the researchers on scientific conferences.

\section{Author affiliations}

${ }^{1}$ Food and Nutrition Science, Department of Biology and Biological Engineering, Chalmers University of Technology, Göteborg, Sweden

${ }^{2}$ Department of Obstetrics and Gynaecology, University of Gothenburg, the

Sahlgrenska Academy, Institute of Clinical Sciences, Göteborg, Sweden

${ }^{3}$ Sunderby Research Unit, Region Norrbotten, Luleå, Sweden

${ }^{4}$ Department of Infectious Diseases, University of Gothenburg, Institute of Biomedicine, the Sahlgrenska Academy, Göteborg, Sweden

${ }^{5}$ Institute of Environmental Medicine, Karolinska Institutet, Stockholm, Sweden

${ }^{6}$ Center of Neurodevelopmental Disorders (KIND), Centre for Psychiatry Research;

Department of Women's and Children's Health, Karolinska Institutet \& Child and 
Adolescent Psychiatry, Stockholm Health Care Services, Stockholm County Council, Stockholm, Sweden

${ }^{7}$ Department of Paediatrics, University of Gothenburg, Institute of Clinical Sciences, Göteborg, Sweden

${ }^{8}$ Department of Odontology/Cariology, Umeå University, Umeå, Sweden ${ }^{9}$ Department of Clinical Sciences, Unit of Pediatrics, Umeå University, Umeå, Sweden

Acknowledgements The authors would like to acknowledge study nurses Marjut Larsson, Ulrika Börlin and study assistant nurse EmmaLundin-Råberger, study midwives Louise Lindgren and Lisa Sundén and study administrator Elvira Sandin for their fantastic work with recruitment, follow-up assessments, sample preparation and data collection. The authors would like to acknowledge Staffan Nilsson, associate professor in mathematical statistics, Mathematical Sciences, Chalmers University of Technology, for performing the power calculations and Carl Brunius, associate professor in Food and Nutrition Science, Department of Biology and Biological Engineering, Chalmers University of Technology for assistance with the section on data analysis. The authors would also like to thank all personnel at the maternity clinics, antenatal clinics and delivery ward for informing and recruiting patients and collecting samples. The authors would like to thank all the families who participated in this study. The authors also gratefully acknowledge our collaborators: Jens Nilsen, professor, Systems Biology, Department of Biology and Biological Engineering, Chalmers University of Technology, Gothenburg, Sweden; Robert Lundqvist, statistician, Research and Innovation Unit, Region Norrbotten; Susanne Lager, PhD, Department of Women's and Children's Health, Uppsala University; Linda Englund-Ögge, MD, PhD, and Maria Hallingström, research midwife, Department of Obstetrics and Gynaecology, Sahlgrenska University Hospital, Gothenburg, Sweden; and Karin Larsson, PhD, and Annette Almgren, research engineer, Food and Nutrition Science, Departments of Biology and Biological Engineering, Chalmers University of Technology, Sweden.

Contributors AS, A-SS and AEW initiated the study and are responsible for the conception and design of the work. All authors have been involved in the design of the cohort and is involved in data analysis and interpretation. FM and AS are responsible for data collection. MB and AR drafted the first version of the manuscript. KB, KJ, MK, NS, MV, AEW, A-SS and AS was involved in writing different parts of the manuscript. AIB, SB, BH, BJ, HR and FS contributed to the critical revision of the manuscript. $M B$ was responsible for finalising the last version of the manuscript. All authors read and approved the manuscript before submission.

Funding Funding has been received from the Swedish Research Council (VR) project no 521-2013-3154, Swedish Research Council for Health, Working Life and Welfare (FORTE) project no 2014-0923, the Västra Götaland Region RUN 612-061815, Åke Wibergs Stiftelse, Jane and Dan Olsson Foundation, the Research and Innovation Unit at Region Norrbotten and through a regional agreement between Umeå University and Västerbotten County Council in the field of Medicine, Odontology and Health (711251).

Competing interests None declared.

Patient consent Not required.

Ethics approval The NICE cohort has been approved by the Regional Ethical Review Board in Umeå, Sweden (2013/18-31M).

Provenance and peer review Not commissioned; externally peer reviewed.

Open access This is an open access article distributed in accordance with the Creative Commons Attribution Non Commercial (CC BY-NC 4.0) license, which permits others to distribute, remix, adapt, build upon this work non-commercially, and license their derivative works on different terms, provided the original work is properly cited, appropriate credit is given, any changes made indicated, and the use is non-commercial. See: http://creativecommons.org/licenses/by-nc/4.0/.

\section{REFERENCES}

1. Berggren S, Roswall J, Alm B, et al. Parents with overweight children two and five years of age did not perceive them as weighing too much. Acta Paediatr 2018;107:1060-4.

2. Wang M, Karlsson C, Olsson C, et al. Reduced diversity in the early fecal microbiota of infants with atopic eczema. J Allergy Clin Immunol 2008;121:129-34.

3. Sandin A, Bråbäck L, Norin E, et al. Faecal short chain fatty acid pattern and allergy in early childhood. Acta Paediatr 2009;98:823-7.

4. Jonsson K, Barman M, Moberg S, et al. Fat intake and breast milk fatty acid composition in farming and nonfarming women and allergy development in the offspring. Pediatr Res 2016;79:114-23.
5. Dietert RR. Developmental immunotoxicity, perinatal programming, and noncommunicable diseases: focus on human studies. Adv Med 2014;2014:1-18.

6. Ahmed S, Moore SE, Kippler M, et al. Arsenic exposure and cellmediated immunity in pre-school children in rural Bangladesh. Toxicol Sci 2014;141:166-75.

7. Barman M, Johansson S, Hesselmar B, et al. High levels of both n-3 and n-6 long-chain polyunsaturated fatty acids in cord serum phospholipids predict allergy development. PLoS One 2013;8:e67920.

8. Gilman SE, Hornig M, Ghassabian A, et al. Socioeconomic disadvantage, gestational immune activity, and neurodevelopment in early childhood. Proc Natl Acad Sci U S A 2017;114:6728-33.

9. Grether JK, Ashwood P, Van de Water J, et al. Prenatal and newborn immunoglobulin levels from mother-child pairs and risk of autism spectrum disorders. Front Neurosci 2016;10:218.

10. Meltzer A, Van de Water J. The role of the immune system in autism spectrum disorder. Neuropsychopharmacology 2017;42:284-98.

11. Vuong HE, Hsiao EY. Emerging roles for the gut microbiome in autism spectrum disorder. Biol Psychiatry 2017;81:411-23.

12. Gardner RM, Kippler M, Tofail F, et al. Environmental exposure to metals and children's growth to age 5 years: a prospective cohort study. Am J Epidemiol 2013;177:1356-67.

13. Kippler M, Tofail F, Hamadani JD, et al. Early-life cadmium exposure and child development in 5-year-old girls and boys: a cohort study in rural Bangladesh. Environ Health Perspect 2012;120:1462-8.

14. Hamadani JD, Tofail F, Nermell B, et al. Critical windows of exposure for arsenic-associated impairment of cognitive function in pre-school girls and boys: a population-based cohort study. Int J Epidemiol 2011;40:1593-604.

15. Skröder H, Kippler M, Tofail F, et al. Early-life selenium status and cognitive function at 5 and 10 years of age in Bangladeshi Children. Environ Health Perspect 2017;125:1552-9924.

16. NCD Risk Factor Collaboration (NCD-RisC). Worldwide trends in body-mass index, underweight, overweight, and obesity from 1975 to 2016: a pooled analysis of 2416 population-based measurement studies in 128.9 million children, adolescents, and adults. Lancet 2017;390:2627-42

17. Källestål C. The effect of five years' implementation of cariespreventive methods in Swedish high-risk adolescents. Caries Res 2005;39:20-6.

18. Strömberg N, Esberg A, Sheng N, et al. Genetic- and Lifestyledependent Dental Caries Defined by the Acidic Proline-rich Protein Genes PRH1 and PRH2. EBioMedicine 2017;26:38-46.

19. Esberg A, Sheng N, Mårell L, et al. Streptococcus mutans adhesin biotypes that match and predict individual caries development. EBioMedicine 2017;24:205-15.

20. Christensen SE, Möller E, Bonn SE, et al. Two new meal- and webbased interactive food frequency questionnaires: validation of energy and macronutrient intake. J Med Internet Res 2013;15:e109.

21. Christensen SE, Möller E, Bonn SE, et al. Relative validity of micronutrient and fiber intake assessed with two new interactive meal- and Web-based food frequency questionnaires. J Med Internet Res 2014;16:e59.

22. Delisle Nyström $\mathrm{C}$, Henriksson $\mathrm{H}$, Alexandrou $\mathrm{C}$, et al. Validation of an Online Food Frequency Questionnaire against Doubly Labelled Water and $24 \mathrm{~h}$ Dietary Recalls in Pre-School Children. Nutrients 2017;9:66.

23. Bonn SE, Trolle Lagerros $\mathrm{Y}$, Christensen SE, et al. Active-Q: validation of the web-based physical activity questionnaire using doubly labeled water. J Med Internet Res 2012;14:e29

24. Bonn SE, Surkan PJ, Lagerros YT, et al. Feasibility of a novel webbased physical activity questionnaire for young children. Pediatr Rep 2012;4:37.

25. Zuriarrain Reyna Y, López Neyra A, Sanz Santiago V, et al. Assessing the measurement of airway resistance by the interrupter technique. Arch Argent Pediatr 2013;111:495-501.

26. Rech VV, Vidal PC, Melo Júnior HT, et al. Airway resistance in children measured using the interrupter technique: reference values. $J$ Bras Pneumol 2008;34:1806-3756.

27. Skin tests used in type i allergy testing position paper. subcommittee on skin tests of the european academy of allergology and clinical immunology. Allergy 1989;44:1-59.

28. Sandin A, Björkstén B, Bråbäck L. Development of atopy and wheezing symptoms in relation to heredity and early pet keeping in a Swedish birth cohort. Pediatr Allergy Immunol 2004;15:316-22.

29. Hesselmar B, Saalman R, Rudin A, et al. Early fish introduction is associated with less eczema, but not sensitization, in infants. Acta Paediatr 2010;99:1861-7.

30. Williams HC, Burney PG, Hay RJ, et al. The U.K. Working Party's Diagnostic Criteria for Atopic Dermatitis. I. Derivation of a 
minimum set of discriminators for atopic dermatitis. $\mathrm{Br} \mathrm{J}$ Dermatol 1994;131:383-96.

31. Williams HC, Burney PG, Pembroke AC, et al. The U.K. Working Party's Diagnostic Criteria for Atopic Dermatitis. III. Independent hospital validation. Br J Dermatol 1994;131:406-16.

32. Williams HC, Burney PG, Strachan D, et al. The U.K. Working Party's Diagnostic Criteria for Atopic Dermatitis. II. Observer variation of clinical diagnosis and signs of atopic dermatitis. $\mathrm{Br} J$ Dermatol 1994;131:397-405.

33. Lundell AC, Johansen S, Adlerberth I, et al. High proportion of CD5+ $B$ cells in infants predicts development of allergic disease. $J$ Immunol 2014:193:510-8.

34. Strömbeck A, Rabe H, Lundell AC, et al. High proportions of FOXP3(+) CD25(high) T cells in neonates are positively associated with allergic sensitization later in childhood. Clin Exp Allergy 2014:44:940-52.

35. Sandin A, Björkstén B, Böttcher MF, et al. High salivary secretory IgA antibody levels are associated with less late-onset wheezing in lgEsensitized infants. Pediatr Allergy Immunol 2011;22:477-81.

36. Savolainen OI, Sandberg AS, Ross AB. A simultaneous metabolic profiling and quantitative multimetabolite metabolomic method for human plasma using gas-chromatography tandem mass spectrometry. J Proteome Res 2016;15:259-65.

37. Soni NK, Ross AB, Scheers $N$, et al. Splenic immune response is down-regulated in c57bl/6j mice fed eicosapentaenoic acid and docosahexaenoic acid enriched high fat diet. Nutrients 2017;9:50.

38. Broberg K, Ahmed S, Engström K, et al. Arsenic exposure in early pregnancy alters genome-wide DNA methylation in cord blood, particularly in boys. J Dev Orig Health Dis 2014;5:288-98.
39. Kippler M, Engström K, Mlakar SJ, et al. Sex-specific effects of early life cadmium exposure on DNA methylation and implications for birth weight. Epigenetics 2013;8:494-503.

40. Masood A, Stark KD, Salem N. A simplified and efficient method for the analysis of fatty acid methyl esters suitable for large clinical studies. J Lipid Res 2005;46:2299-305.

41. Barman $\mathrm{M}$, Jonsson $\mathrm{K}$, Sandin $\mathrm{A}$, et al. Serum fatty acid profile does not reflect seafood intake in adolescents with atopic eczema. Acta Paediatr 2014;103:968-76.

42. Barman M, et al. Serum levels of vitamin a and atopic rhinoconjunctivitis in swedish adolescents. Journal of Food Science and Nutrition Therapy 2017;3:014-19.

43. Barman M, Jonsson $\mathrm{K}$, Hesselmar $\mathrm{B}$, et al. No association between allergy and current 25 -hydroxy vitamin $D$ in serum or vitamin $D$ intake. Acta Paediatr 2015;104:405-13.

44. Lu Y, Kippler M, Harari F, et al. Alkali dilution of blood samples for high throughput ICP-MS analysis-comparison with acid digestion. Clin Biochem 2015;48:140-7.

45. Rydbeck F, Bottai M, Tofail F, et al. Urinary iodine concentrations of pregnant women in rural Bangladesh: a longitudinal study. J Expo Sci Environ Epidemiol 2014;24:504-9.

46. Frenck-Mestre C, Besson M, Pynte J. Finding the locus of semantic satiation: an electrophysiological attempt. Brain Lang 1997;57:406-22.

47. Sjöberg F, Barkman C, Nookaew I, et al. Low-complexity microbiota in the duodenum of children with newly diagnosed ulcerative colitis. PLoS One 2017;12:e0186178.

48. Hanhineva K, Brunius C, Andersson A, et al. Discovery of urinary biomarkers of whole grain rye intake in free-living subjects using nontargeted LC-MS metabolite profiling. Mol Nutr Food Res 2015;59:2315-25. 